\section{Aberrant FOXP3 gene expression in eutopic and ectopic endometrium of infertile women with endometriosis}

\author{
Gustavo Mendonça \\ Andre, \\ Fernanda Abani Mafra, \\ Viviane Cavalcanti, \\ Tatiana Guida Ponce, \\ Denise Maria \\ Christofolini, \\ Bianca Bianco, \\ Caio Parente Barbosa
}

\title{
Abstract
}

Background: Immunological theories suggest that changes in the immune system could prevent the ability to eliminate the endometrium of the pelvic cavity. In women with endometriosis is possible that changes in immunity mediated by $T$ cells facilitate the implantation of endometrial fragments or cells in ectopic locations and recent studies have associated the FOXP3 gene with homeostasis of the immune system and the development of autoimmune diseases. We aimed to evaluate the expression of FOXP3 gene in both eutopic and ectopic endometrium of infertile women with endometriosis and controls.

Methods: A case-control study was performed comprising 25 infertile women with endometriosis and 44 fertile women without endometriosis. FOXP3 and GAPDH expression was measured by mRNA using quantitative reverse transcription polymerase chain reaction (qRT-PCR) based on TaqMan methodology. The Mann-Whitney test was used to compare the values between the groups.

Results: The results disclosed that mean expression of FOXP3 in eutopic endometrium of endometriosis group was significantly higher when compared to the control group $(p=0,008)$, regardless the stage of the disease. Considering the samples of the ectopic endometrium, FOXP3 expression was also significantly higher in endometriosis group compared to the control group $(p=0,004)$, regardless the stage of the disease.

1 Human Reproduction and Genetics Center - Faculdade de Medicina do $A B C$, Santo André/SP, Brazil.

Contact information:

\section{Bianca Bianco.}

Faculdade de Medicina do ABC.

Tel-Fax: (55) 1 4993-5464

Address: 4400 Avenida Príncipe de Gales, 821 - Santo André/SP, CEP 09060-650, Brasil.

झ bianca.bianco@hotmail.com 
Conclusion: The results of this study point to an association between the expression of FOXP3 and the genesis/progression of endometriosis

Keyword: Autoimmunity; Endometrium; Endometriosis; Infertility; FOXP3 gene.

\section{Introduction}

Endometriosis is a common gynecological disease, defined as the growth of endometrial tissue outside the uterine cavity that often results in dyspaurenia, dysmenorrhea, pelvic pain and infertility [1,2]. It affects $3-10 \%$ of women in their reproductive years and $20-50 \%$ of women with infertility $[2,3]$.

Numerous hypotheses have been put forward to explain the presence of ectopic endometrial tissue and stroma. Immunological theories suggest that changes in the immune system could prevent the ability to eliminate the endometrium of the pelvic cavity $[4,5]$. In women with endometriosis is possible that changes in immunity mediated by $T$ cells facilitate the implantation of endometrial fragments or cells in ectopic locations $[6,7]$. The immune cells which are likely to play roles in this destruction, including cells such as macrophages, natural killer [NK] and cytotoxic T-cells must be tightly regulated in order to ensure that the immune response is specific to sloughed endometrial fragments and not the intact uterine tissue. The cells which are almost certainly the key regulators of this response are a distinct population of T-cells, known as regulatory T-cells, called Tregs $[8,9]$.

Recent studies have associated the FOXP3 gene (Gene ID: 50943, Xp11.23) with homeostasis of the immune system and the development of autoimmune diseases $[8,10,11]$. The FOXP3 gene is primarily expressed in CD4+ CD25+ Tregs in normal physiological conditions. It encodes FOXP3 protein which regulates the activation of $T$ cell and functions as a transcriptional repressor and down-regulates cytokine production in T cells [8,12-14].
The human endometrium is a tissue with high cell turnover and marked cyclical remodeling under the influence of ovarian steroid hormones. During the normal menstrual cycle the endometrium within the uterus is widely infiltrated by immune cells. The specific activities of these immune cells are crucial for the proper course of such reproductive processes as menstruation and implantation [15]. An alteration in Treg lymphocyte infiltration generally disrupts the immunological equilibrium [16]. The increase in the number of Treg cells depends upon estrogen levels, and estrogens are also responsible for the increase in the immune suppressive potential of these cells [17]. Estrogen levels generally seem to be linked with a decrease in $\mathrm{TH} 1$ response and the absence of Treg cell fluctuation can be linked to an immune defect arising with the development of endometriosis $[16,18]$.

Considering the complex cellular and molecular mechanisms involved in endometriosis formation and progression, and that immune regulators are likely to play crucial roles in diseases within which numerous immune factors appear to be highly disturbed, we aimed to verify a possible relationship between FOXP3 expression and the development/ progression of endometriosis.

\section{Methods}

The Subjects: Among the patients of the Human Reproduction and Genetics Center of the Faculdade de Medicina do ABC, Santo André, Brazil, 25 inferti-

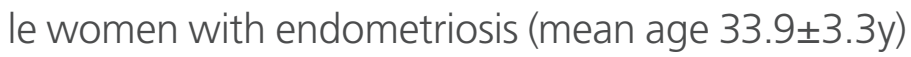
were selected to take part in this study. They were 
diagnosed with endometriosis by laparoscopy and classified according to the American Society for Reproductive Medicine (19), with obligatory histological confirmation of the disease. Patients with acute or chronic disorders, especially autoimmune diseases, were excluded. In this group, minimal/mild (stage I and II) endometriosis was found in 15 cases $(60.0 \%)$, and moderate/severe (stage III and IV) endometriosis in 10 cases (40.0\%).
Considering the characteristics of these patients such as mean age, duration of infertility, body mass index $\left(\mathrm{Kg} / \mathrm{m}^{2}\right)$, value of CA125 (miliUl/mL), value of FSH (miliUl/mL), LH (miliUl/mL), progesterone (ng/ $\mathrm{mL}$ ), prolactin $(\mathrm{ng} / \mathrm{mL})$, presence of chronic pelvic pain, tubal obstruction and male factor infertility compared to the endometriosis stage are described in Table 1. The surgical indication for all patients was a complaint of infertility.

Table 1: Characteristics of studied infertile patients with endometriosis.

\begin{tabular}{|c|c|c|c|}
\hline Characteristics & Minimal/Mild & Moderate/Severe & P-value \\
\hline N & 15 & 10 & \\
\hline Age (Years) & $33.4(3.58)$ & $34.44(3.57)$ & 0.090 \\
\hline Duration of Infertility (Years) & $4.58(3.84)$ & $4.11(3.25)$ & 0.278 \\
\hline BMI (Kg/m²) & $23.67(2.26)$ & $25.46(3.26)$ & 0.006 \\
\hline CA125 (miliUl/mL) & $34.35(32.98)$ & $51.98(36.86)$ & $<0.001$ \\
\hline FSH (miliUl/mL) & $6.03(1.89)$ & $6(1.88)$ & 0.478 \\
\hline LH (miliUl/mL) & $6.69(10.55)$ & $5.06(4.67)$ & 0.088 \\
\hline Progesterone $(\mathrm{ng} / \mathrm{mL})$ & $10.38(6.73)$ & $9.13(10.18)$ & 0.146 \\
\hline Prolactine $(\mathrm{ng} / \mathrm{mL})$ & $16.49(6.04)$ & $16.37(8.26)$ & 0.456 \\
\hline Presence of Pevic Pain & $10 / 15(66 \%)$ & $5 / 10(50 \%)$ & 0.680 \\
\hline Tubal Obstruction & $4 / 15(26 \%)$ & $4 / 10(40 \%)$ & 0.791 \\
\hline Male Factor & $2 / 15(13 \%)$ & $4 / 10(40 \%)$ & 0.292 \\
\hline BMI-Body Mass Index $\quad$ CA125-Cancer Antigen 125 & FSH-Follicle-stimulating & LH-Luteinizing Hormone \\
\hline
\end{tabular}

A control group of 44 fertile women (mean age $35.3 \pm 4.6$ y) was selected especially at the Family Planning Outpatient Clinic of the Faculdade de Medicina do $A B C$ among a group submitted to tubal ligation. In all of them, absence of endometriosis was confirmed by inspection of the pelvic cavity during the laparoscopy. None of these women had any personal and/or familial history of autoimmune diseases.

Moreover, all women (cases and controls) had regular menstrual cycles (25-35 days) and did not use any hormone therapy for at least three months before surgery.
The investigation into the cause of infertility included a hormonal and biochemical profile, testing for sexually transmitted diseases, imaging examinations, investigation of genetic and/or immunological abnormalities, hysterosalpingography, hysteroscopy and laparoscopy, and semen analysis of the partner. Patients with endometriosis who did not achieve pregnancy, at least, after twelve natural or induced cycles following laparoscopy were considered infertile.

Clinical data, peripheral blood and endometrial biopsies samples were collected only after explai- 
ning the objectives of the study and obtaining signed informed consent, as approved by the Faculdade de Medicina do ABC Research Ethics Committee (No.095/2009).

Biopsies: Samples were collected at the luteal phase of the menstrual cycle (21 \pm 2 days). Eutopic endometrium samples were collected with the aspiration cannula (Pipelle $®$, Prodimed, France) and typical endometriotic lesions only of the peritoneum (ectopic endometrium) were collected during laparoscopy/laparotomy. The fragments of the biopsies were immediately placed in an extender solution of RNA (RNA holder, BioAgency, Brazil) and subsequently stored in a freezer at $-80^{\circ} \mathrm{C}$.

RNA extraction: RNA extraction of endometrial biopsies and endometriotic lesions was carried out with Qiazol Lysis Reagent, according to the manufacturer's instructions (Qiagen $®$, Turnberry Lane, CA, USA).

To measure the quantity and quality (purity) of RNA extracted, the extracted sample was measured in NanoDrop 2000 spectrophotometer (Thermo Scientific, CA/USA). The quality/purity of the material was measured by the ratio $230 / 260$ and 260/280.

cDNA synthesis: The cDNA synthesis was done from total RNA using the QuantiTect Reverse Transcription Kit, according to the manufacturer's instructions (Qiagen $®$, Valencia, California, United States).

FOXP3 gene expression: The expression of FOXP3 (ID: 50943 - Hs01085834_m1) and GAPDH (ID 2597-Hs99999905_m1) genes was measured by qRT-PCR, based on the TaqMan methodology using the equipment StepOne Real-Time PCR System (Life Technologies ${ }^{\circledR}$, Foster City, CA, EUA).

$P C R$ reactions were processed to a final volume of $25 \mu \mathrm{L}$, containing $12.5 \mu \mathrm{L}$ of $2 \mathrm{X}$ TaqMan Universal PCR Master Mix (Life Technologies $\circledR_{\text {, Foster }}$ City, CA, USA), $1.25 \mu \mathrm{L}$ TaqMan assay (20x), $1 \mu$ l of sample CDNA, and $10.25 \mu \mathrm{L}$ of RNAse-free water. The PCR conditions were those recommended by the manufacturer: initial denaturation at $50^{\circ} \mathrm{C}(2$ min) to initiate enzyme activity AmpErase UNG, 95 ${ }^{\circ} \mathrm{C}$ (10 min) to initiate the activity of the enzyme AmpliTaq Gold DNA Polymerase, followed by 40 cycles of denaturation at $95^{\circ} \mathrm{C}$ (15 sec) and annealing/extension at $60^{\circ} \mathrm{C}(1 \mathrm{~min})$.

The amount of FOXP3 mRNA from the control group was compared to the respective amounts of patients with endometriosis. The GAPDH gene was used as normalizer of the reactions. The results were analyzed by $\Delta \Delta C$ t method. (20)

Statistical analyses: Statistical analysis was performed using Stata 11.0. For comparison of quantitative variables such as age, duration of infertility, body mass index, value of CA125, amount of FSH, LH, progesterone and prolactin, t test was used, with results expressed as mean and standard deviation. To compare qualitative variables between groups such as the presence of chronic pelvic pain, presence of tubal and male factor infertility, compared to the staging of endometriosis, chi-square test was used.

The comparison between the values of FOXP3 mRNA in the control group and the group of patients with endometriosis was performed using the Mann-Whitney. Due to non-normal distribution of the FOXP3 expression, we chose to describe the data by percentile values $(25,50$ and 75$)$ and the mean and confidence interval of 95\%. A p-value $<0.05$ was considered statistically significant.

\section{Results and Discussion}

The average of FOXP3 expression found in both eutopic and ectopic endometrium of infertile women with endometriosis and controls were shown

\section{in Table 2.}

Considering the endometrium of the women studied, the mean expression of FOXP3 was significantly higher in the endometrium of endometriosis group than in control group $(p=0.008)$, even when the expression of FOXP3 was analyzed in endome- 
Table 2: Values of central tendency and dispersion of FOXP3 expression found in infertile women with endometriosis and controls.

\begin{tabular}{|c|c|c|c|c|}
\hline \multirow[t]{2}{*}{ Studied Group } & \multicolumn{2}{|l|}{ Eutopic Endometrium $(n=25)$} & \multicolumn{2}{|l|}{ Ectopic Endometrium ( $n=25)$} \\
\hline & $\begin{array}{c}\text { FOXP3 expression } \\
\text { P50 (P25 - P75) } \\
\text { Mean (IC95\%) }\end{array}$ & $p^{*}$ & $\begin{array}{c}\text { FOXP3 expression } \\
\text { P50 (P25 - P75) } \\
\text { Mean (IC95\%) }\end{array}$ & $p^{*}$ \\
\hline Endometriosis & $0,54(0,34-1,04) 2,57(0,51 ; 4,64)$ & 0,008 & $1,54(1,03-2,77) 40,32(-2,83 ; 83,47)$ & 0,004 \\
\hline Minimal/Mild $(n=15)$ & $0,54(0,34-1,04) 2,26(0,25 ; 4,27)$ & 0,007 & $1,71(1,08-11,08) \quad 42,11(-13,92 ; 98,15)$ & 0,003 \\
\hline Moderate/Severe $(n=10)$ & $0,46(0,23-2,06) 3,05(-1,80 ; 7,90)$ & 0,002 & $1,29(0,46-2,35) 37,62(-44,56 ; 119,80)$ & 0,007 \\
\hline Control Group $(n=44)$ & $0,99(0,55-1,65)$ & & $1,48(0,99 ; 1,98)$ & \\
\hline \multicolumn{3}{|c|}{ P50, P25, P75: Percentile 50, 25 and 75.} & \multicolumn{2}{|l|}{ IC (95\%): Confidence Interval of 95\%. } \\
\hline
\end{tabular}

triosis minimal/mild and moderate/severe separately. Regarding the samples of peritoneal endometrium (ectopic), FOXP3 expression was significantly higher in endometriosis group compared to the control group ( $\mathrm{p}=0.004)$, even when the expression of FOXP3 was analyzed in endometriosis minimal/mild and moderate/severe separately.

The Treg cells comprise two distinct populations: natural Tregs (nTreg), which originate in the thymus and are specific to self-antigens presented by epithelial thymic cells, and induced Tregs, which are generated back into the periphery from T CD4+ (Tconv) cells through antigenic stimulation in the presence of TGF $\beta$ and foreign antigens [21]. Both populations of Treg lymphocytes express FOXP3, a transcription factor critical for homeostasis and the suppressor function of Tregs [10-12].

Considering the critical role of Treg cells in impairing autoimmunity and previous results relating genetic polymorphisms in the FOXP3 gene to autoimmune diseases, we aimed to evaluate the FOXP3 expression in both eutopic and ectopic endometrium of infertile women with endometriosis. The results disclosed that the mean expression of FOXP3 in the eutopic endometrium of endometriosis group was significantly higher when compared to the control group, regardless the stage of the disease. Considering the samples of the ectopic endometrium, FOXP3 expression was significantly higher compared to the control group, especially in minimal/mild disease.

Berbic et al (2010) [9] showed that the density of peripheral FOXP3+ cells increases during the follicular phase, reaching its peak during the late proliferative phase, when serum estradiol levels are also elevated. It has been proposed that under normal conditions a pre-ovulatory rise in FOXP3+ cells may be required for the induction of immune tolerance required to facilitate successful embryo implantation, should it occur [15]. The failure to down-regulate FOXP3+ expression during the secretory phase in women with endometriosis may well be attributed to increased presence of endometrial antigens, as well as to continuous local estrogen production $[22,23]$ both of which are probably stimulating continuous FOXP3+ cell proliferation in endometriosis.

In contrast, primary unexplained infertility has been associated with reduced expression of FOXP3 mRNA in endometrial tissue in mid-secretory phase of the menstrual cycle [24], suggesting that impaired recruitment of Treg cells, or insufficient differentiation of uterine T-cells into Treg cells even prior to conception may affect the capacity to establish pregnancy in women. 
André et al (2011) [25] aimed to evaluate FOXP3 polymorphisms (rs3761549, rs3761548, rs2232368, rs2232366 and rs2280883) in a group of infertile women with and without endometriosis and controls. The single-marker analysis revealed that FOXP3 rs3761549 was significantly associated with endometriosis $(p=0.003)$, regardless of the stage of the disease. Considering the infertile group without endometriosis, single-marker analysis revealed statistical difference for rs2280883 ( $p=0.024)$ and rs2232368 $(p=0.034)$ FOXP3 polymorphisms. No associations were found considering rs3761548 and rs2232366 either for endometriosis-related infertility group or idiopathic infertility group. Haplotype analysis of five FOXP3 polymorphisms identified a haplotype "CTTGA" associated with endometriosis ( $p=0.011$ ) and also identified a haplotype "ACTAG" that was associated with idiopathic infertility $(p=0.014)$. After Bonferroni correction, only the rs3761549 polymorphism associated with endometriosis remains statistically significant, strengthening the association of this polymorphism with the disease. The authors concluded that FOXP3 polymorphisms can be associated with risk of idiopathic infertility (rs2280883 and rs2232368) and endometriosis (rs3761549) in Brazilian women.

Moreover, recently our group [26] demonstrated a cumulative effect of two genetic polymorphisms (FOXP3 C-2383T/rs3761549 and FCRL3 C$169 T /$ rs7528684) those were previously shown to be associated with endometriosis. The combined genotypes of FCRL3 and FOXP3 polymorphisms showed a positive association between genotypes FCRL3TT/FOXP3CT, FCRL3CT/FOXP3CT and FCRL3CC/FOXP3CT and the risk of endometriosis development. Besides, a progression of the disease risk was observed according to the presence of one or two copies of risk allele FCRL3 $C$ and only one copy of risk allele FOXP3 T (OR=2.14, OR=3.25 and $\mathrm{OR}=6.0$, respectively, for genotypes $F C R L 3 T T /$ FOXP3CT, FCRL3CT/FOXP3CT and FCRL3CC/
FOXP3CT), suggesting a possible gene-gene interaction leading to a cumulative effect on endometriosis development.

Utilizing the induced non-human primate (Papio anubis) model of endometriosis, Braundmeier et al (2012) [27] showed that, in control animals, the proportion of peripheral natural Tregs (nTregs) was reduced $(P<0.05)$ during the mid- and late secretory stages of the menstrual cycle compared with menses. The induction of disease decreased peripheral Treg expression at early time points $(P<0.05)$ and this remained low throughout the time course, compared with the pre-inoculatory level of an individual. FOXP3 gene expression and Treg populations were also decreased in the eutopic endometrium $(\mathrm{P}<0.05)$ compared with control animals, whereas these parameters were increased in ectopic lesions $(P<0.05)$, compared with the eutopic endometrium, suggesting that a reduction in peripheral Tregs may be a causative factor for endometriosis-associated infertility, while the increase in ectopic Treg expression may aid lesion development. Furthermore, endometriosis appears to disrupt Treg recruitment in both eutopic and ectopic endometrium. As Braundmeier et al (2012) [27], we found that, in human, FOXP3 is aberrant expressed in both eutopic and ectopic endometrium of infertile women with endometriosis, strengthening the association of FOXP3 and the genesis/progression of endometriosis.

Chen et al (2012) [28] aimed to investigate the pathogenesis of infertility in women with endometriosis by comparing FoxP3+ T regulatory cells expression in the eutopic endometrium of infertile women with endometriosis $(n=27)$ and endometrium from healthy fertile women $(n=20)$ in peri-implantation phase by quantitative real-time RT-PCR and FoxP3 protein expression was assessed by immunohistochemistry. Similarly with our findings they observed that FoxP3 mRNA expression in all infertile patients with endometriosis was significantly higher than the control group. Further analysis based on the extent 
of the disease revealed that FoxP3 mRNA expression in infertile patients with advanced endometriosis was significantly higher than the mild endometriosis group and the control group. Immunohistochemistry analysis showed predominant positive staining for FoxP3 protein in the endometrial stroma.

Podgaec et al [29] also in 2012 evaluated CD4+CD25highFoxp3+ cells and IL-6, IL-10, IL-17, and TGFb in the peritoneal fluid of women with endometriosis $(n=70)$ and controls $(n=28)$ using flow cytometry abd RT-PCR. The results disclosed that the lymphocytes in the peritoneal fluid of women with endometriosis were higher CD4+CD25high compared to the control group. Foxp3 expression was similarly elevated in patients with the disease compared to those without. IL-6 and TGF-b were also higher in endometriosis group and IL-10 and IL-17 showed no significant differences between the two groups. The authors concluded that peritoneal fluid of patients with endometriosis had a higher percentage of CD4+CD25highFoxp3+ cells and also higher levels of IL- 6 and TGF-b compared to women without the disease, suggesting that CD4+CD25highFoxp3+ cells may play a role in the pathogenesis of endometriosis.

Since retrograde menstruation is a common phenomenon that occurs in the majority of women during their reproductive life, this fact alone is not likely to be responsible for the onset of endometriosis. It is believed that intrinsic properties of the endometrial tissue are responsible for ensuring viability, adhesion, neovascularization and establishment of ectopic lesions in women with endometriosis [30].

Endometriosis has been widely characterized as an inflammatory disease. Numerous studies have shown that peritoneal leukocytes and their inflammatory mediators exert local effects, creating a microenvironment that may facilitate the development and progression of these lesions. The immune system appears to be significantly deregulated not only at the injury site, but also in the uterine cavity of women with endometriosis $[4,30]$.

\section{Conclusion}

It is important to point out that Tregs are responsible for both beneficial and deleterious effects. Although Tregs prevent excessive inflammatory and autoimmune responses, they also suppress necessary immunity and, therefore, also need regulations to restrict their effects.

In conclusion, our data point to an association between the expression of FOXP3 and the genesis/ progression of endometriosis.

\section{Conflict of Interest}

The authors declare no conflict of interest

\section{Acknowledgments}

The authors wish to thank CNPq for granting Bianca Bianco (300825/2013-7), Caio Parente Barbosa (300816/2012-0) and Denise Maria Christofolini (301242/2013-5) a research productivity scholarship.

\section{Authors' Contributions}

Gustavo Andre, Caio Parente Barbosa and Bianca Bianco conceived study design. Gustavo Andre, Fernanda Mafra, Viviane Cavalcanti and Tatiana Ponce performed the data collection and analysed data. Gustavo Andre, Fernanda Mafra, Caio Parente Barbosa, Denise Maria Christofolini and Bianca Bianco interpretation the data. All authors were involved in literature search, writing the paper and had final approval of the submitted and published versions. The authors have no competing interests 


\section{References}

1. Barbosa $C P$, de Souza AM, Bianco B, Christofolini D, Mafra FA, Lima GR: OC-125 immunostaining in endometriotic lesion samples. Arch Gynecol Obstet 2010, 281: 43-7.

2. Giudice LC, Kao LC: Endometriosis. Lancet 2004, 364: 1789-99.

3. Barbosa CP, Souza AM, Bianco B, Christofolini D, Mafra FA, Lima GR: Frequency of endometriotic lesions in peritoneum samples from asymptomatic fertile women and correlation with CA125 values. Sao Paulo Med J 2009, 127: 342-5.

4. Matarese G, De Placido G, Nikas Y, Alviggi C: Pathogenesis of endometriosis: natural immunity dysfunction or autoimmune disease? Trends Mol Med 2003, 9: 223-8.

5. Antsiferova YS, Sotnikova NY, Posiseeva LV, Shor AL: Changes in the T-helper cytokine profile and in lymphocyte activation at the systemic and local levels in women with endometriosis. Fertil Steril 2005, 84: 1705-11.

6. Dmowski WP, Gebel HM, Braun DP: The role of cell-mediated immunity in pathogenesis of endometriosis. Acta Obstet Gynecol Scand Suppl 1994, 159: 7-14.

7. Bianco B, André GM, Vilarino FL, Peluso C, Mafra FA, Christofolini DM, Barbosa CP: The possible role of genetic variants in autoimmune-related genes in the development of endometriosis. Hum Immunol 2012, 73(3): 306-15.

8. Vignali DA, Collison LW, Workman CJ: How regulatory T cells work. Nat Rev Immunol 2008, 8: 523-32.

9. Berbic M, Hey-Cunningham AJ, Ng C, Tokushige N, Ganewatta S, Markham R, et al: The role of Foxp3+ regulatory T-cells in endometriosis: a potential controlling mechanism for a complex, chronic immunological condition. Hum Reprod 2010, 25: $900-7$.

10. Ban Y, Tozaki T, Tobe T, Ban Y, Jacobson EM, Concepcion ES, et al: The regulatory T cell gene FOXP3 and genetic susceptibility to thyroid autoimmunity: an association analysis in Caucasian and Japanese cohorts. J Autoimmun 2007, 28: 201-7.

11. Gao L, Li K, Li F, Li H, Liu L, Wang L, et al: Polymorphisms in the FOXP3 gene in Han Chinese psoriasis patients. J Dermatol Sci 2010, 57: 51-56.
12. McHugh RS, Whitters MJ, Piccirillo CA, Young DA, Shevach EM, CollinsM, et al: CD4(+)CD25(+) immunoregulatory T cells: gene expression analysis reveals a functional role for the glucocorticoid-induced TNF receptor. Immunity 2002, 16: 31123.

13. Hori S, Nomura T, Sakaguchi S: Control of regulatory T cell development by the transcription factor Foxp3. Science 2003, 299: 1057-61.

14. Ziegler SF. FOXP3: of mice and men. Annu Rev Immunol 2006, 24: 209-26.

15. Arruvito L, Sanz M, Banham AH, Fainboim L: Expansion of CD4+, CD25+ and FOXP3+ regulatory T cells during the follicular phase of the menstrual cycle: implications for human reproduction. J Immunol 2007, 178: 2572-2578.

16. Basta P, Majka M, Jozwicki W, Lukaszewska E, Knafel A, Grabiec $M$, et al: The frequency of $C D 25+C D 4+$ and FOXP3+ regulatory $T$ cells in ectopic endometrium and ectopic decidua. Reprod Biol Endocrinol 2010, 8: 116.

17. Polanczyk MJ, Hopke C, Vandenbark AA, Offner H: Treg suppressive activity involves estrogen-dependent expression of programmed death-1 (PD-1). Int Immunol 2007, 19: 337-343.

18. Saito S, Nakashima A, Shima T, Ito M: Th1/Th2/Th17 and regulatory T-cell paradigm in pregnancy. Am J Reprod Immunol 2010, 63: 601-610.

19. Revised American Society for Reproductive Medicine classification of endometriosis. Fertil Steril 1997, 67: 817-21.

20. Livak KJ, Schmittgen TD: Analysis of relative gene expression data using real-time quantitative PCR and the 2(-Delta Delta C(T)) Method. Methods 2001, 25(4): 402-8.

21. Liston A, Rudensky AY: Thymic development and peripheral homeostasis of regulatory T cells. Curr Opin Immunol 2007, 19: $176-85$.

22. Prieto $G A$, Rosenstein $Y$ : Oestradiol potentiates the suppressive function of human CD4+ CD25+ regulatory $T$ cells by promoting their proliferation. Immunology 2006, 118: 58-65.

23. Tai $P$, Wang J, Jin $H$, Song $X$, Yan J, Kang $Y$, et al: Induction of regulatory $\mathrm{T}$ cells by physiological level estrogen. J Cell Physiol 2008, 214: 456-64. 
24. Jasper MJ, Tremellen KP, Robertson SA: Primary unexplained infertility is associated with reduced expression of the T-regulatory cell transcription factor Foxp3 in endometrial tissue. Mol Hum Reprod 2006, 12: 301-308.

25. André GM, Barbosa CP, Teles JS, Vilarino FL, Christofolini DM, Bianco B: Analysis of FOXP3 polymorphisms in infertile women with and without endometriosis. Fertil Steril 2011, 95: 2223-7.

26. Barbosa CP, Teles JS, Lerner TG, Peluso C, Mafra FA, Vilarino FL, Christofolini DM, Bianco B: Genetic association study of polymorphisms FOXP3 and FCRL3 in women with endometriosis. Fertil Steril 2012, 97(5): 1124-8.

27. Braundmeier A, Jackson K, Hastings J, Koehler J, Nowak R, Fazleabas A: Induction of endometriosis alters the peripheral and endometrial regulatory $T$ cell population in the non-human primate. Hum Reprod 2012, 27(6): 1712-22

28. Chen S, Zhang J, Huang C, Lu W, Liang Y, Wan X: Expression of the $T$ regulatory cell transcription factor FoxP3 in periimplantation phase endometrium in infertile women with endometriosis. Reprod Biol Endocrinol 2012, 27; 10: 34.

29. Podgaec S, Rizzo LV, Fernandes LF, Baracat EC, Abrao MS: CD4(+) CD25(high) Foxp3(+) cells increased in the peritoneal fluid of patients with endometriosis. Am J Reprod Immunol 2012, 68(4): 301-8.

30. Taylor RN, Lebovic DI, Mueller MD: Angiogenic factors in endometriosis. Ann NY Acad Sci 2002, 955: 89-100.

\section{Comment on this article:}
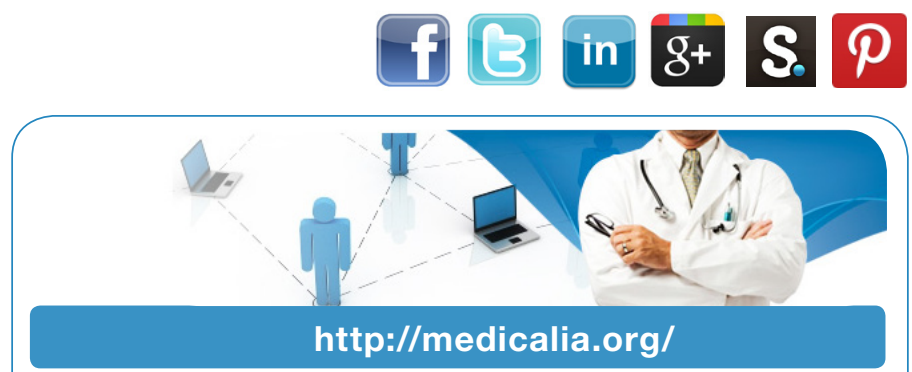

Where Doctors exchange clinical experiences, review their cases and share clinical knowledge. You can also access lots of medical publications for free. Join Now!

\section{Publish with iMedPub}

\section{http://www.imed.pub}

International Archives of Medicine is an open access journal publishing articles encompassing all aspects of medical science and clinical practice. IAM is considered a megajournal with independent sections on all areas of medicine. IAM is a really international journal with authors and board members from all around the world. The journal is widely indexed and classified Q1 in category Medicine. 